\title{
Use of an Intracranial Drain as a Conduit for Treatment of an Intracranial Streptococcus intermedius Abscess
}

\author{
Shoeb B. Lallani ${ }^{1}$, Melanie Hyte ${ }^{2}$, Emily Trieu ${ }^{3}$, Carlos Reyes-Sacin ${ }^{4}$, Ninh Doan ${ }^{5}$ \\ 1. Neurology, University of Alabama at Birmingham School of Medicine, Birmingham, USA 2. Pharmacology, Baptist \\ Medical Center South, Montgomery, USA 3. Neurosurgery, Pleasant Grove High School, Elk Grove, USA 4. Infectious \\ Disease, Baptist Medical Center South, Montgomery, USA 5. Department of Neurosurgery, Medical College of \\ Wisconsin, Milwaukee, USA
}

Corresponding author: Shoeb B. Lallani, slalla2@uab.edu

\begin{abstract}
Brain abscesses are difficult to manage clinically and often result in a poor outcome. Although surgical and medical therapeutics have progressed, there are still challenges that make treating intracranial abscesses problematic. One of these treatment barriers is the poor penetration of intravenous antibiotics to the infection source through the blood-brain barrier. In this case report, we will discuss the use of a surgical drain as a conduit for direct antibiotic administration for a rare, recurrent Streptococcus intermedius infection. This technique allows us to bypass the blood-brain barrier while also reducing the systemic effects of antibiotics. When used in conjunction with craniotomy and resection, direct antibiotic administration via a surgical drain proved to be effective at treating our patient's abscess and preventing recurrence.
\end{abstract}

Categories: Infectious Disease, Neurosurgery

Keywords: intracranial abscess, microbiology, infectious disease, antibiotic administration, neurosurgery

\section{Introduction}

Intracranial abscess is a devastating diagnosis with a high rate of morbidity and mortality that affects a wide range of populations. Even with the advent of new technologies and approaches to target these abscesses, there remains a $10 \%$ mortality rate within the general population $[1,2]$. One of the difficulties with current methods for treatment of intracranial abscesses is proper access to the lesion site. It can often be difficult to achieve adequate levels of antibiotics at the lesion via an intravenous introduction without causing systemic toxicity [3]. In this article, we explore a case study employing an intracranial drain as a conduit for direct drug administration in the treatment of a rare Streptococcus intermedius infection.

Review began 04/08/2021 Review ended 04/16/2021 Published 04/21/2021

\section{() Copyright 2021} Lallani et al. This is an open access article distributed under the terms of the Creative Commons Attribution License CC-BY 4.0., which permits unrestricted use, distribution, and reproduction in any medium, provided the original author and source are credited.

\section{Case Presentation}

A 70-year-old female presented to her primary care physician complaining of headaches. Her primary care physician gave her an initial diagnosis of sinusitis and prescribed her an intramuscular corticosteroid injection, but her headache continued to worsen. Over the next week, the patient stopped talking, eating, and developed right-sided weakness. Due to this progression of symptoms, magnetic resonance imaging (MRI) of the brain was ordered which revealed multiple cerebral ring-enhancing lesions, with the largest lesion measuring $4.6 \mathrm{~cm}$ in the right frontal lobe white matter. There was a $3.6 \mathrm{~cm}$ lesion in the left parietal lobe white matter and another $3.6 \mathrm{~cm}$ lesion in the left occipital lobe. All lesions demonstrated surrounding vasogenic edema, local mass effect, increased internal T2 signal, and restricted diffusion (Figure 1). The patient was taken to surgery for craniotomy and resection of the frontal and left parietal abscesses. Specimens for culture were taken intraoperatively. Final culture was positive for $S$. intermedius, sensitive to ceftriaxone (minimum inhibitory concentration of $0.12 \mu \mathrm{g} / \mathrm{mL})$, clindamycin $(0.06 \mu \mathrm{g} / \mathrm{mL})$, levofloxacin $(0.5$ $\mu \mathrm{g} / \mathrm{mL})$, linezolid $(1.0 \mu \mathrm{g} / \mathrm{mL})$, and penicillin $(0.03 \mu \mathrm{g} / \mathrm{mL})$. 


\section{Cureus}

A

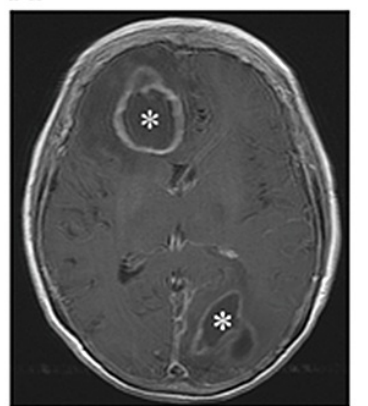

$\mathrm{E}$

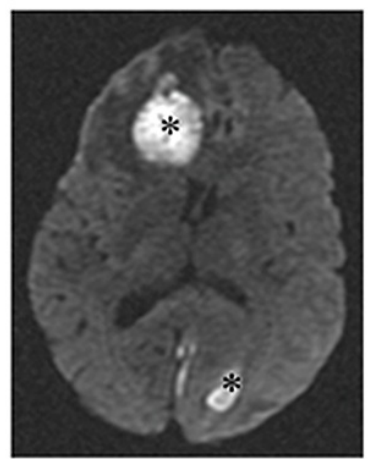

$\mathrm{B}$

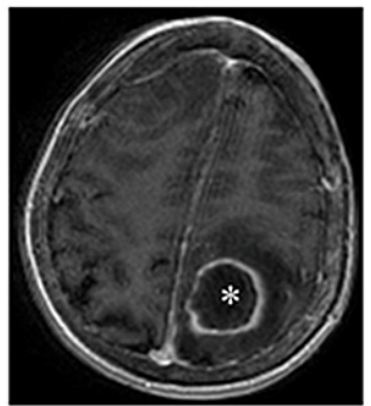

$\mathrm{F}$

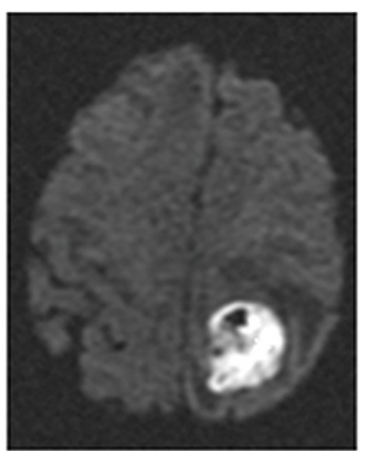

$\mathrm{C}$

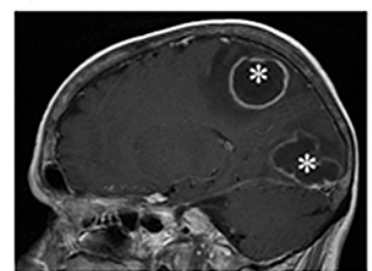

$\mathrm{D}$

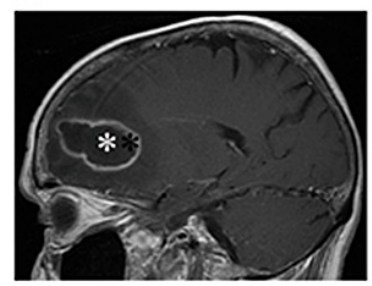

\section{FIGURE 1: Initial MRI with contrast.}

Initial MRI of the brain with contrast (A-D) and DWI (E-F) showing the three large enhancing lesions in frontal, occipital, and parietal areas with DWI signals consistent with the presence of brain metastasis or abscesses $(*)$.

MRI: magnetic resonance imaging; DWI: diffusion-weighted imaging

Her mentation improved after the surgery and she was discharged to an extended care facility where she was treated with $2 \mathrm{~g}$ of intravenous ceftriaxone every 12 hours for six weeks. While at the facility, she was noted to be febrile and demonstrated a decline in mentation by the house staff. She was transported back to the emergency department, where she was found to be lethargic and globally aphasic, with worsening rightsided weakness, a fever of $101.9^{\circ} \mathrm{F}$, and an elevated white blood cell count of $29.6 \times 10^{3}$. Computed tomography (CT) and MRI of the brain with and without contrast showed a new, large epidural abscess along the superior interhemispheric fissure between the frontal and parietal lobes, measuring $20 \mathrm{~mm}$ in thickness. There was also worsening of the multifocal cerebral abscess in the left parieto-occipital lobe (Figures 2, 3). 


\section{Cureus}

A

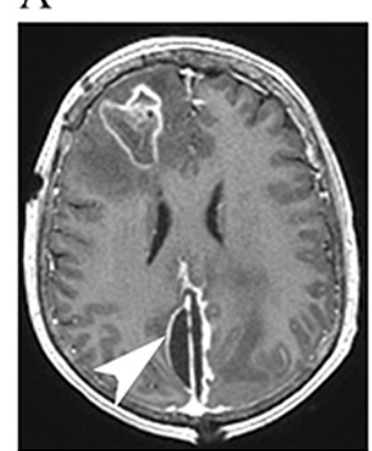

$\mathrm{D}$

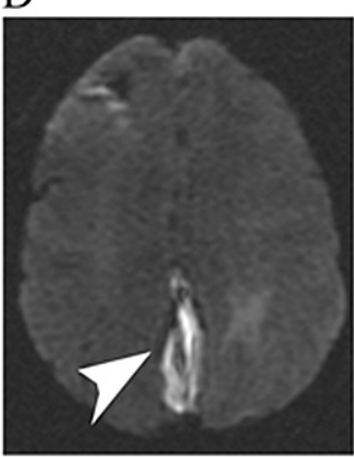

B

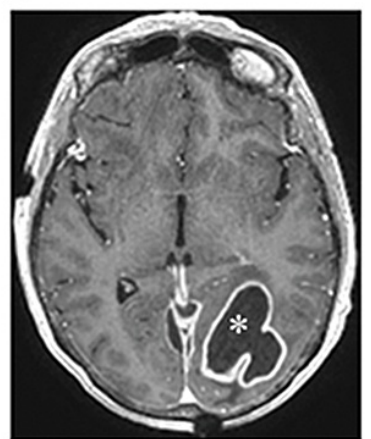

E

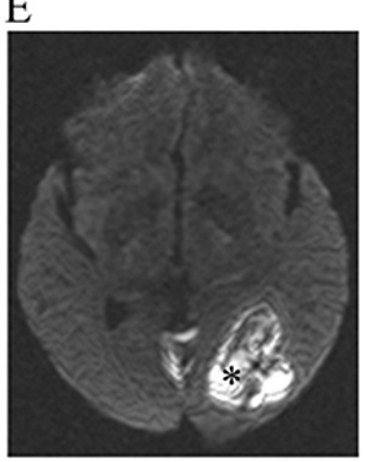

$\mathrm{C}$

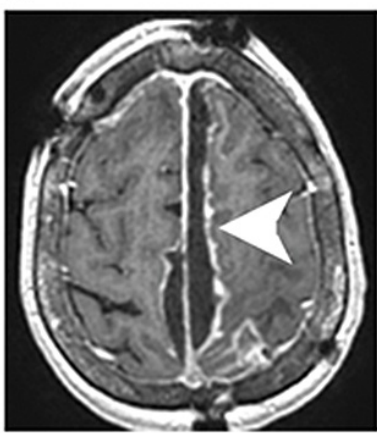

$\mathrm{F}$

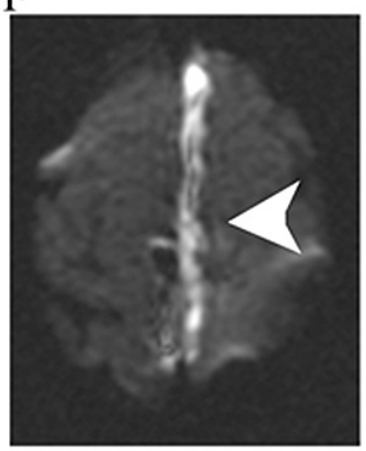

FIGURE 2: Repeat MRI with contrast T1.

MRI of the brain axial with contrast T1 sequence (A-C) and DWI sequence (D-F) with new subdural empyema (arrows) and worsening of the occipital abscess are shown, suspected of communications between these lesions.

MRI: magnetic resonance imaging; DWI: diffusion-weighted imaging 


\section{Cureus}

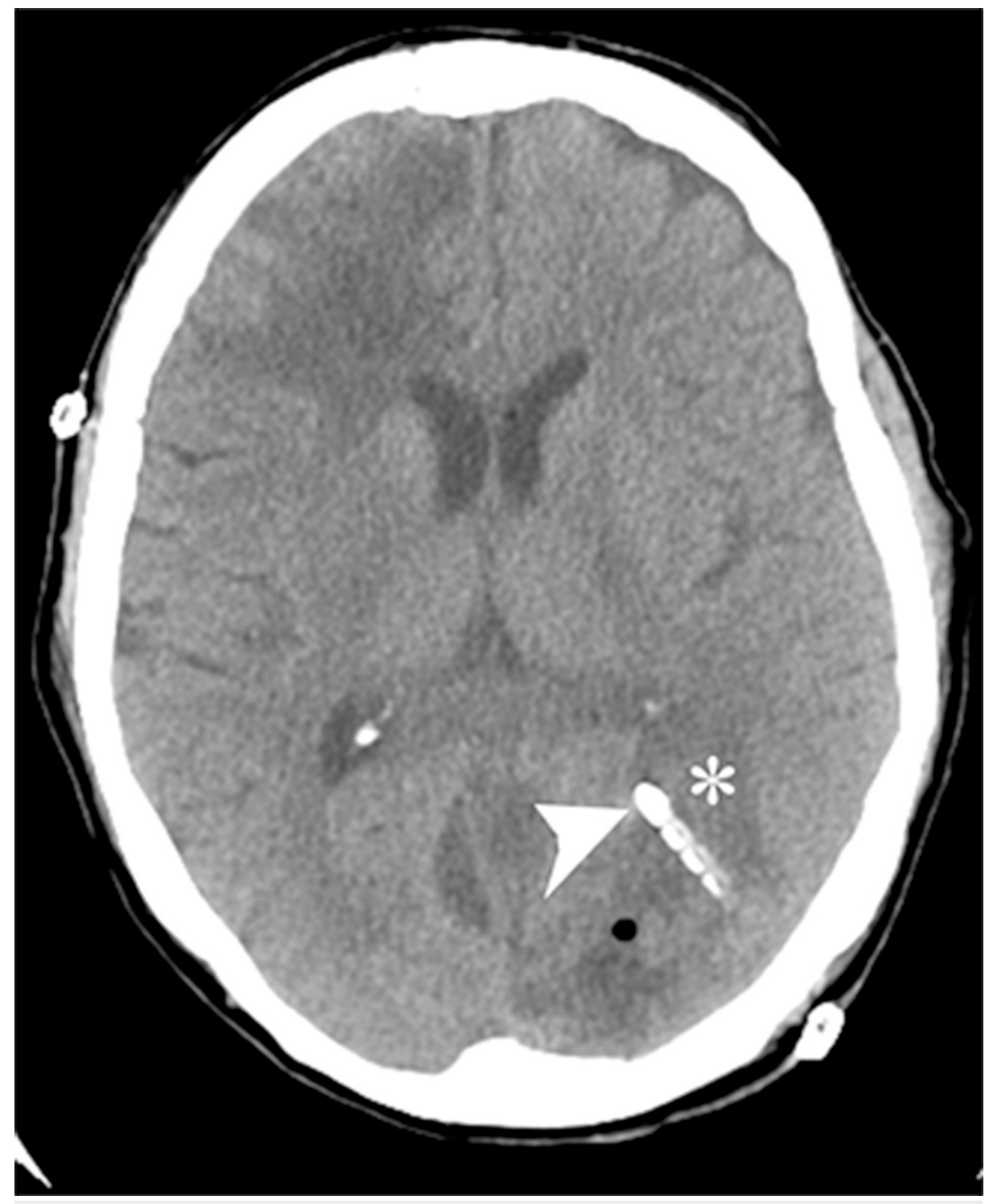

FIGURE 3: CT brain without contrast showing abscess and catheter.

CT of the brain without contrast is shown with the abscess $\left({ }^{*}\right)$ penetrated by the catheter (arrow), through which vancomycin was injected for direct intra-abscess antibiotic treatment.

CT: computed tomography

The patient was taken to the operating room for stereotactic drainage of the left subdural abscess, hydrogen peroxide irrigation, and drain placement in the left occipital abscess. Surgical culture showed $S$. intermedius once again. Post-operatively, $10 \mathrm{mg}$ of vancomycin $(2 \mathrm{~mL}$ of $5 \mathrm{~g} / \mathrm{mL}$ solution) once a day was administered directly into the left occipital abscess through the drain daily for three days, along with $2 \mathrm{~g}$ of intravenous ceftriaxone every 12 hours for six weeks. At the three-month follow-up, MRI demonstrated a complete resolution of the abscess and subdural empyema without obvious neurological deficit (Figure 4). 


\section{Cureus}

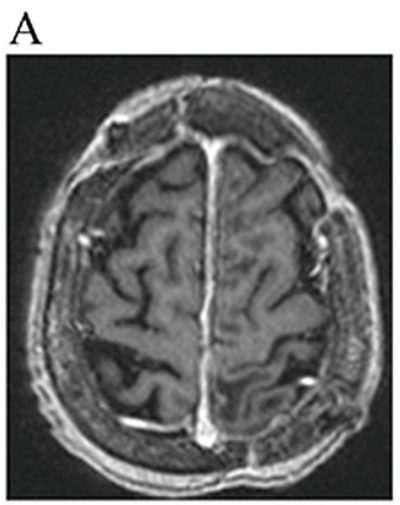

$\mathrm{B}$

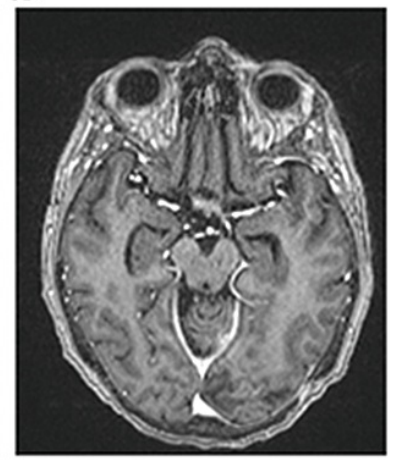

$\mathrm{C}$

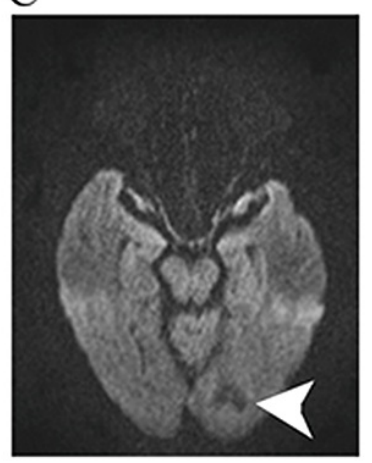

FIGURE 4: Three-month follow-up MRI.

Three-month follow-up MRI of the brain with contrast (A-B) and DWI sequence (C) illustrating no abnormal enhancement of DWI signal (arrow) in the area corresponding the to prior subdural empyema and the abscess indicating subdural empyema, and the occipital abscess are completely resolved.

MRI: magnetic resonance imaging; DWI: diffusion-weighted imaging

\section{Discussion}

Although intracranial abscesses are rare $(0.9$ per 100,000$)$ [4], they continue to be a life-threatening condition. Early recognition is paramount to proper treatment and prevention of neurological compromise. However, presenting signs and symptoms of abscess are often nonspecific and vary greatly depending on the size and location of the lesion. One of the most common symptoms reported with brain abscesses is headaches, followed by altered awareness, fever, seizure, and focal neurological deficits [5]. The origin of some brain abscesses can be traced to previous maxillofacial infections, including sinusitis and mastoiditis or prior craniotomy; however, the etiology of most infections remains uncertain [6].

The majority of these abscesses result from infection due to gram-positive bacteria, most often Staphylococcus aureus and S. milleri [7]. Current empiric treatment of intracranial abscesses involves surgical resection of the lesion followed by intravenous broad-spectrum antibiotics, covering for gram-positive, gram-negative, and anaerobic bacteria [8]. However, there has been a progressive increase in the number of drug-resistant bacteria infecting brain parenchyma, resulting in higher rates of recurrence following initial intervention. These resistant organisms often require vancomycin and linezolid for adequate treatment [9].

S. intermedius is a common co-infector of intracranial abscesses, but rarely is it the predominant species [7]. When $S$. intermedius has been found to be the predominant bacteria in a brain abscess, it is usually secondary to hematogenous spread from a prior focus of infection (e.g., sinus, teeth, middle ear), most often seen in children [10]. However, the origin of $S$. intermedius intracranial abscesses in adults has historically been difficult to identify, as is the case with our patient [11]. In addition to the rarity of the predominance of this species in brain abscesses, studies have shown that $S$. intermedius has developed resistance to many commonplace antibiotics, including penicillin, cephalosporins, macrolides, ciprofloxacin, and clindamycin. Fortunately, there are antibiotics that have been shown to be consistently effective against $S$. intermedius such as vancomycin, teicoplanin, and imipenem $[12,13]$.

Similar to other studies, our patient did not have a clear origin for her $S$. intermedius infection, with no history of recent infection that could have contributed to the formation of her intracranial abscess. Our patient did not respond entirely to her initial treatment with intravenous ceftriaxone and had a recurrence of her abscess. A possibility for the recurrence of the infection is due to inadequate levels of the drug at the target intracranial site. Studies have shown that ceftriaxone has decreased penetrability through the bloodbrain barrier when there is a lack of meningeal inflammation, making it difficult to achieve adequate levels of the antibiotic in the brain parenchyma [14]. As the patient did not respond to standard intravenous antibiotic treatment and her health was deteriorating after primary intervention, we decided to treat her recurrence with vancomycin via an intracranial drain while continuing the intravenous treatment. The use of an intracranial drain as a conduit for drug administration has been shown to be a safe and viable option in other studies in the past [15]. We chose to use vancomycin rather than ceftriaxone as intrathecal administration of vancomycin has been well studied while the outcomes of intrathecal administration of ceftriaxone are not well known [16]. Furthermore, our initial study showed that the organism was susceptible to vancomycin, making this an appropriate choice. The combination of systemic and targeted therapy proved to be effective in our case as our patient showed resolution of her symptoms without recurrence. 


\section{Conclusions}

Brain abscesses can be challenging to diagnose early and have a complicated treatment regimen. Most cases are due to $S$. aureus and $S$. milleri, but here we presented a rare case of intracranial abscess caused by $S$. intermedius. Furthermore, due to the failure of traditional intravenous ceftriaxone and recurrence of the abscess, we used an uncommon technique of targeting the lesion site by administering vancomycin through a surgical drain. The combination of systemic and direct targeting of the intracranial abscess proved to be effective in the treatment of our patient's recurrent infection.

\section{Additional Information \\ Disclosures}

Human subjects: Consent was obtained or waived by all participants in this study. Conflicts of interest: In compliance with the ICMJE uniform disclosure form, all authors declare the following: Payment/services info: All authors have declared that no financial support was received from any organization for the submitted work. Financial relationships: All authors have declared that they have no financial relationships at present or within the previous three years with any organizations that might have an interest in the submitted work. Other relationships: All authors have declared that there are no other relationships or activities that could appear to have influenced the submitted work.

\section{References}

1. Beach JE, Perrott J, Turgeon RD, Ensom MHH: Penetration of vancomycin into the cerebrospinal fluid: a systematic review. Clin Pharmacokinet. 2017, 56:1479-90. 10.1007/s40262-017-0548-y

2. Bahubali VKH, Vijayan P, Bhandari V, Siddaiah N, Srinivas D: Methicillin-resistant Staphylococcus aureus intracranial abscess: an analytical series and review on molecular, surgical and medical aspects. Indian J Med Microbiol. 2018, 36:97-103. 10.4103/ijmm.IJMM_17_41

3. Alvis Miranda H, Castellar-Leones SM, Elzain MA, Moscote-Salazar LR: Brain abscess: current management. J Neurosci Rural Pract. 2013, 4:67-81. 10.4103/0976-3147.116472

4. Lu CH, Chang WN, Lin YC, et al.: Bacterial brain abscess: microbiological features, epidemiological trends and therapeutic outcomes. QJM. 2002, 95:501-9. 10.1093/qjmed/95.8.501

5. Sharma R, Mohandas K, Cooke RP: Intracranial abscesses: changes in epidemiology and management over five decades in Merseyside. Infection. 2009, 37:39-43. 10.1007/s15010-008-7359-x

6. Patel K, Clifford DB: Bacterial brain abscess. Neurohospitalist. 2014, 4:196-204. 10.1177/1941874414540684

7. Mishra AK, Fournier PE: The role of Streptococcus intermedius in brain abscess . Eur J Clin Microbiol Infect Dis. 2013, 32:477-83. 10.1007/s10096-012-1782-8

8. Roche M, Humphreys H, Smyth E, et al.: A twelve-year review of central nervous system bacterial abscesses; presentation and aetiology. Clin Microbiol Infect. 2003, 9:803-9. 10.1046/j.1469-0691.2003.00651.x

9. Kumari VH, Babu AR, Srinivas D, Siddaiah N, Somanna S: Methicillin-resistant Staphylococcus aureus central nervous system infections--analysis and outcome. Br J Neurosurg. 2015, 29:413-8. 10.3109/02688697.2015.1006168

10. Vajramani GV, Nagmoti MB, Patil CS: Neurobrucellosis presenting as an intra-medullary spinal cord abscess . Ann Clin Microbiol Antimicrob. 2005, 4:14. 10.1186/1476-0711-4-14

11. Yamamoto M, Fukushima T, Ohshiro S, Go Y, Tsugu H, Kono K, Tomonaga M: Brain abscess caused by Streptococcus intermedius: two case reports. Surg Neurol. 1999, 51:219-22. 10.1016/s0090-3019(97)00505-3

12. Jacobs JA, Stobberingh EE: In-vitro antimicrobial susceptibility of the "Streptococcus milleri' group (Streptococcus anginosus, Streptococcus constellatus and Streptococcus intermedius). J Antimicrob Chemother. 1996, 37:371-5. 10.1093/jac/37.2.371

13. Limia A, Jiménez ML, Alarcón T, López-Brea M: Five-year analysis of antimicrobial susceptibility of the Streptococcus milleri group. Eur J Clin Microbiol Infect Dis. 1999, 18:440-4. 10.1007/s100960050315

14. Nau R, Sörgel F, Eiffert H: Penetration of drugs through the blood-cerebrospinal fluid/blood-brain barrier for treatment of central nervous system infections. Clin Microbiol Rev. 2010, 23:858-83. 10.1128/CMR.00007-10

15. Doan N, Nguyen H, Luyuan L, Shabani S, Gelsomino M, Johnson V: Good outcomes with the intraventricular vancomycin therapy in a patient with ruptured brain abscesses. Asian J Neurosurg. 2018, 13:396-9. 10.4103/1793-5482.185065

16. Inamasu J, Kuramae T, Tomiyasu K, Nakatsukasa M: Fulminant ependymitis following intraventricular rupture of brain abscess. J Infect Chemother. 2011, 17:534-7. 10.1007/s10156-010-0201-2 